\title{
Mechanical Changes in Crab Nerve Fibers during Action Potentials
}

\author{
Ichiji TASAKI, Kunihiko IwaSA, and Robert C. GibBons \\ Laboratory of Neurobiology, National Institute of Mental Health, \\ Bethesda ,Maryland, U.S.A. and Marine Biological Laboratory, \\ Woods Hole, Massachusetts, U.S.A.
}

\begin{abstract}
A nerve impulse travelling along a crustacean nerve was found to be accompanied by a small, rapid movement of the nerve surface. The movement was $10-20 \mathrm{~nm}$ in amplitude and was concurrent with a rise in the "swelling pressure" of the order of $5 \mathrm{mg} / \mathrm{cm}^{2}$ for a nerve bundle. Initiation of an action potential at the site of cathodal polarization was preceded by a small, slow mechanical change in the nerve fiber. Anodal polarization produced a large mechanical change of the opposite sign. Tetrodotoxin and procaine suppressed rapid mechanical changes.
\end{abstract}

Several previous investigators suggested the existence of rapid mechanical changes in nerve fibers concomitant with the production of action potentials (KAYUSHIN and LyUdKovsKaya, 1955; FrANK, 1958; LetTVIN et al., 1962; Hill et al., 1977). The alleged rapid mechanical changes reported before signal averages became available were all very large and most of those findings could not be confirmed by subsequent investigators (SANDLIN et al., 1968; CoHEN, 1973). Hill et al. (1977) employed the laser interferometry method combined with signal averaging and reported that electric excitation of a crayfish nerve fiber induced a rapid reduction of the fiber diameter followed by a relaxation.

The present paper describes the results of our demonstration of small and rapid mechanical changes in crab nerve fibers during action potentials by the use of both optical and mechano-electric methods. We found that there is a distinct upward movement of a light-reflecting target placed on the surface of a nerve when action potentials are generated by electric stimulation. Furthermore, we could demonstrate that this movement is produced by a transient swelling of the nerve fiber. Obviously, these rapid mechanical changes are totally unrelated to the slow mechanical changes (KornaKova et al., 1947; HiLl, 1950; BRYANT and ToBIAS, 1955) which are known to be produced cumulatively by a large number of action potentials.

In the present paper, we deal exclusively with the results obtained by using

Received for publication May 29, 1980 
crustacean nerve fibers; the results obtained from squid giant axons will be described later. Preliminary accounts of our results obtained with the same methods are presented elsewhere (TASAKI and IWASA, 1980; IWASA and TASAKI, 1980).

\section{METHODS}

Most of the experiments described below were carried out on claw nerves of the blue crab, Collinectes sapidus. On several occasions, nerves taken from walking legs or claws of the lobster (Homarus americanus) or claw nerves of the crayfish (Procambarus clarkii) were also used for comparison; no clear difference in behavior was observed among different preparations of crustacean nerves. The procedure of dissecting claw nerves of the crab is described elsewhere (TASAKI and Sisco, 1975). Electron microscopic examination of the claw nerve of the blue crab indicated that the fiber diameter ranges between 1 and $12 \mu \mathrm{m}$. The connective tissue and the blood vessels attached to the bundle of these nerve fibers were removed under a dissecting microscope prior to the following examination of mechanical properties of the fibers.

Nerve chambers employed were designed to enhance the mechanical stability of the portion of the nerve used for measurements. The bottom surface of the chamber was usually constructed in a convex, semicylindrical form, so that the lower surface of the nerve under tension was pressed against the chamber bottom. A pair of stimulating electrodes $(\mathrm{Pt}$ or $\mathrm{Ag}$ ) were placed near one end of the nerve in the chamber; a pair of extracellular recording electrodes were arranged usually near the other end.

The standard optical probe used in the present studies was a Fotonic sensor purchased from Mechanical Technology, Inc., Latham, N.Y. This probe consists of two bundles of optic fibers mixed at one end. One of the two bundles was used for transmitting strong white light from a source (Osram quartz-iodine lamp) to the mixed (i.e., sensing) end; the other bundle was employed for carrying light from the sensing end to a photo-detector (Pin-10, United Detector Technology, Inc., Santa Monica, Ca). To measure small displacements of the surface of a nerve, a light-reflecting target was placed above the nerve. Gold dust, a piece of aluminum foil, a small piece of platinum wire, a small ball of silver or stone, etc. were used for this purpose; no significant difference was observed in the results obtained with different kinds of targets. Diagram A, in Fig. 1, shows the dependence of the observed light intensity upon the distance between the target and the sensing end of the Fotonic sensor employed. The output of the operational amplifier (Analog Devices, Norwood, Mass.) connected to the photo-detector rises sharply with the distance and reaches a maximum at about $150 \mu \mathrm{m}$. As the distance is increased further, there is a gradual fall in the intensity. After determining the position of the maximum intensity for each target employed, the tip of the sensor was moved to one of the roughly linear portions of the intensitydistance curve, either on the "proximal" or "distal" side of the maximum. 

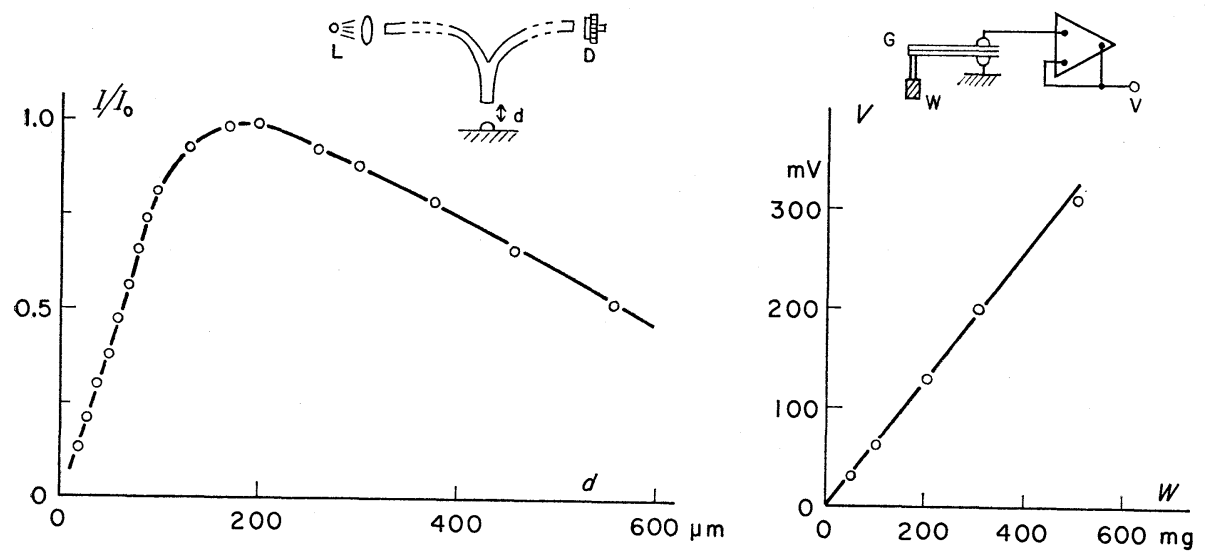

Fig. 1. Calibration of Fotonic sensor (left) and Gulton bender (right). The diagrams at the top show the experimental setups schematically (see text). The maximum value of the light intensity, $I_{0}$, observed with the Fotonic sensor was 2-4 $\mu \mathrm{A}$. L stands for light source; D, detector; d, distance between light-reflecting target and sensor; G, Gulton bender; W, weight; V, voltage-output of bender.

Small movements of the target associated with the production of action potentials are calculated by the following linear formula:

$$
\Delta l=\Delta I /\left(s_{i} I_{0}\right),
$$

where $s_{i}$ is the slope of the light intensity-distance curve, $\Delta I$ the amplitude of the change in light intensity associated with the production of action potentials, and $I_{0}$ the maximum value of the light intensity observed. The tip of the sensor was usually placed at the position of the steepest rise; this "proximal" slope was approximately $(115 \mu \mathrm{m})^{-1}$ when the target was a piece of short platinum wire. To supplement the data taken at the proximal slope, the position of a linearly declining portion of the intensity-distance curve was also used; the slope of the curve at this "distal" position was approximately $-(770 \mu \mathrm{m})^{-1}$. We usually measured the displacement at $I / I_{0}=0.6$ on the proximal slope and at $I / I_{0}=0.7$ on the distal slope. These points are located near the centers of the linear ranges. It is important to note that we can distinguish up-and-down motion of the target from other modes of target motion by comparing the mechanical records taken at the two different slopes. We used this comparison to check our results.

The standard mechano-electric transducer employed in the present investigation was a piezo-ceramic bender (model R050S) kindly supplied by Gulton Industries, Inc., Methuchen, N.J. An approximately $6 \mathrm{~mm}$-long bender was used in conjunction with a voltage-follower made with an FET operational amplifier (Analog Devices, Norwood, Mass.). The sensitivity of the bender was calibrated by hanging known weights from the end of the bender (see Diagram B, in Fig. 1). The voltage-sensitivity of the bi-morph bender was roughly $0.5 \mathrm{mV} / \mathrm{mg}$. To test 
whether or not the bender can follow rapid changes in tension, the tip of the bender was driven sinusoidally by connecting to a calibrated condenser-microphone by means of a thin thread. We found that the bender could follow up to about $10,000 \mathrm{~Hz}$. In the range below $1,000 \mathrm{~Hz}$, there was no distinct peak of resonance.

To measure pressure change, a stylus was installed close to the free end of the bender, which was mounted on a micromanipulator. A pressure was applied to the nerve surface through the stylus. A detailed description is given in an earlier report (IWASA and TASAKI, 1980).

The voltage-output of the FET operational amplifier connected to the Fotonic sensor or to the piezo-ceramic bender was amplified by a factor of 1,000 with a condenser-coupled Tektronix preamplifier. The output of the preamplifier was displayed directly on the screen of a Tektronix oscilloscope (model 502). Under the conditions of the experiments described below, the signal-to-noise ratio at the output of the preamplifier was of the order of 2-10. For a quantitative determination, therefore, signal-averaging over 4-152 trials was adopted.

\section{RESULTS}

1. Small rapid movement of the surface of nerve fibers associated with propagated impulse

In preliminary studies, we found that there is a small, transient, upward movement of a small piece of platinum placed on the nerve when an impulse arrives at the site of mechanical recording. In crab nerves, the magnitude of the movement was in the range between 10 and $20 \mathrm{~nm}$. We made optical targets of various materials, insulators as well as conductors, and obtained consistent results. In those studies, the site of mechanical recording was only a few $\mathrm{mm}$ away from the site of electric stimulation. In the following studies, an attempt was made to determine the velocity at which the locus of the maximum movement travels along the nerve.

The diagram on the top of Fig. 2 illustrates the arrangement of the two sites of mechanical recording in relation to the stimulating and recording electrodes. The entire 35-40 mm-long desheathed nerve was immersed in seawater. The light-reflecting targets were placed at two sites along the nerve, one at a distance of $5 \mathrm{~mm}$ (proximal target) and the other at $15 \mathrm{~nm}$ (distal target) away from the site of stimulation. The separation between the stimulating and the recording electrodes was $24 \mathrm{~mm}$. The stimulating current pulses were $0.3 \mathrm{msec}$ in duration and the strength employed was approximately 30 times the threshold value.

As expected, the mechanical record taken at the distal target had a latency longer than that obtained at the proximal target. The velocity of the mechanical response determined by this procedure was roughtly $4 \mathrm{~m} / \mathrm{sec}$ at $21^{\circ} \mathrm{C}$. This value was slightly smaller than the velocity of the fastest dominant peak of the action potential, approximately $5 \mathrm{~m} / \mathrm{sec}$. This slight difference may be attributed to a 
A
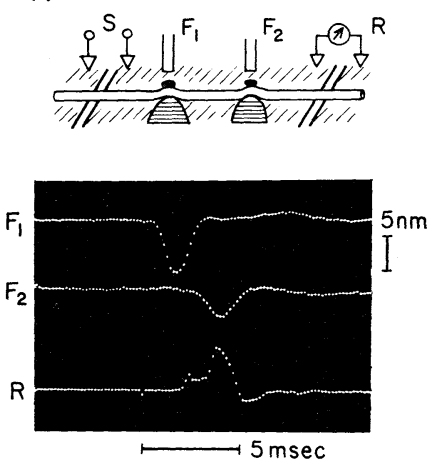

B
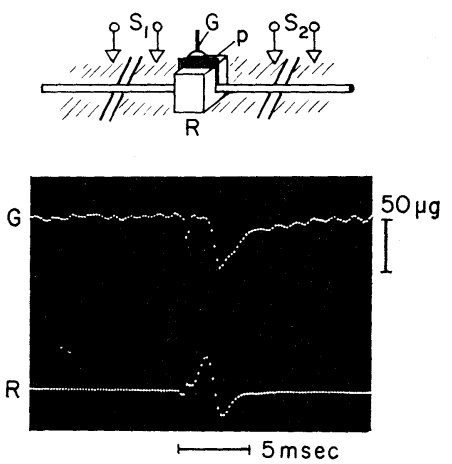

Fig. 2. A) Top: Diagram illustrating the arrangement of the stimulating electrodes (S), two sites of mechanical recording $\left(F_{1}\right.$ and $\left.F_{2}\right)$ and the position of the electrodes used for recording and the action potential $(\mathrm{R})$. Bottom: Mechanical and electrical responses obtained with the setup illustrated above. $21^{\circ} \mathrm{C}$. B) Demonstration of swelling of a crab nerve associated with the arrival of nerve impulses at the site of mechanical recording from two sides. $S_{1}$ and $S_{2}$ represent stimulating electrodes located at the same distance from the recording electrode $R$ at the botton of the slot. $G$ represents a stylus attached to a Gulton bender pressing the nerve by the mediation of a thin plastic plate, $\mathrm{P}$, located above the recording electrode $\mathrm{R}$. In the record shown at the bottom, the upper trace represents the mechanical response recorded with the Gulton bender, $\mathrm{G}$, and the lower trace the action potential observed with the recording electrode, $\mathrm{R}$. The downward deflection of the mechanical trace represents a rise in the pressure $22^{\circ} \mathrm{C}$.

greater contribution of small nerve fibers to the mechanical responses. The amplitude of the mechanical response recorded at the distal target was smaller and longer in duration than those taken at the proximal target. This difference is attributable to an increase in temporal dispersion of nerve impulses in individual fibers associated with nerve conduction.

\section{Increase in diameter of nerve fibers during action potential}

We have seen in a preliminary experiment that there is a transient rise in the pressure exerted by a nerve on a stylus on the nerve when an action potential is generated. The surface area of the stylus used was relatively small (approximately $0.1 \mathrm{~mm}^{2}$ ). The possibility could not be excluded therefore that the observed mechanical response was simply a sign of a localized movement of the membrane surface without being accompanied by a change in the volume of individual nerve fibers. The following observations were made with a view to determining whether or not the mechanical responses are accompanied by a change in the volume occupied by nerve fibers.

A nerve chamber provided with a special narrow slot in the middle was used (see Fig. $1 \mathrm{~B}$, top). The slot was about $7 \mathrm{~mm} \mathrm{long}$, roughly $0.7 \mathrm{~mm}$ wide at the bottom, and approximately $5 \mathrm{~mm}$ deep. The upper portion of the slot was slightly 
wider so that a desheathed nerve trunk could be introduced into the slot without difficulty. A thin plastic plate (approximately $0.6 \mathrm{~mm}$ in thickness and $7 \mathrm{~mm}$ in width) was introduced above the nerve in the slot (see P in the figure). Finally, a force of $100-500 \mathrm{mg}$ was applied to the nerve by means of a Gulton bender (G) pressing the plastic plate. Under these conditions, the space within the slot is packed with nerve fibers; therefore, swelling of individual nerve fibers during an action potential is expected to raise the pressure exerted by the nerve on the bender.

The electric response was recorded with a fine silver electrode, $\mathrm{R}$ in the figure, at the bottom of the slot (referring to the electrode immersed in seawater surrounding the slot). The bottom of the slot was usually covered with a thin (roughly $2 \mathrm{~mm}$ thick) layer of cotton soaked with seawater. The nerve fibers were excited on both sides of the slot so that two nerve impulses reached the slot simultaneously from the two sides of the fibers. The use of this arrangement was intended to prevent the plastic plate $(7 \mathrm{~mm}$ wide) in the slot from tilting upon arrival of a propagated pressure change from one side.

The mechanical record, in Fig. 2B, top, indicates that there was a definite rise in the pressure at the time of arrival to two nerve impulses. The small difference in latency between the electrical and mechanical responses may be attributed, at least partly, to the inertia and the friction of the mechanical recording system. It seems reasonable therefore to conclude, from these observations, that the cross-sectional area of the nerve fibers increases when action potentials are generated in the fibers.

\section{Effects of electric currents}

It is well known that a long pulse of electric current produces histological and optical changes in nerve fibers (see BETHE, 1920; CoHEN, 1973). Therefore, it is not surprising to find that mechanical changes in nerve fibers can be generated by application of electric currents.

We employed the following experimental arrangement to examine the effects of electric currents on mechanical properties of crab nerves. A desheathed nerve was introduced into a plastic chamber consisting of a $6 \mathrm{~mm}$-wide middle pool of seawater separated from large lateral pools by $8 \mathrm{~mm}$-wide air-gaps. In order to prevent dessication, the portion of the nerve passing through the air-gap was completely covered with vaseline. By means of a large silver wire electrode, the middle pool was grounded. The two lateral pools were shunted electrically by means of silver electrodes. Pulses of constant currents were applied between the middle and lateral pools, and the mechanical changes of the portion of the nerve in the middle pool was detected either with a Fotonic sensor or with a Gulton piezo-ceramic bender.

Two examples of the mechanical records obtained by the optical method are presented in Fig. 3. When the applied current was directed inwards through the 


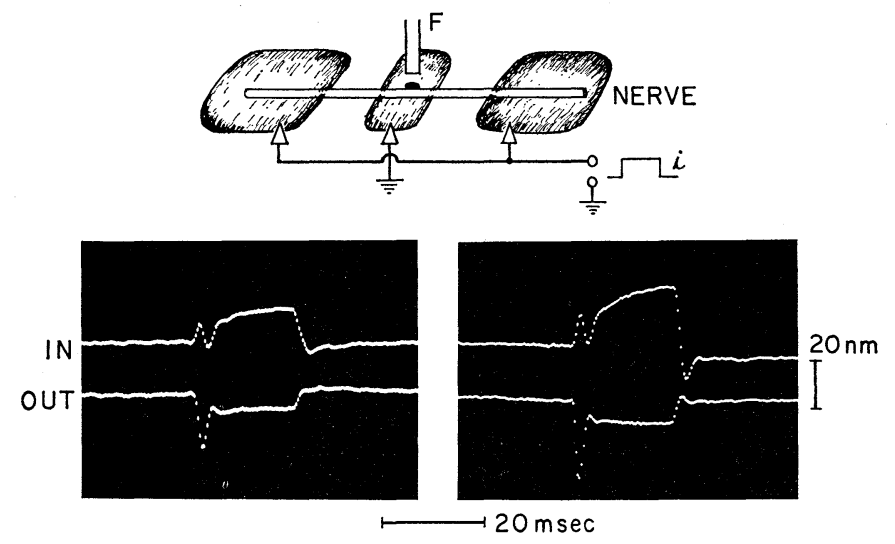

Fig. 3. Mechanical responses produced by long current pulses. The diagram at the top illustrates the experimental setup employed (see text). The upper trace marked IN was obtained with a current traversing inward through the nerve membrane in the middle pool (namely, with a hyperpolarizing current). The trace marked OUT shows the record obtained with an outwardly directed current (namely, with a depolarizing current). The upward deflection of the Fotonic sensor output represents an increase in the distance between the light-reflecting target on the nerve and the sensor. The sharp, downward deflection of the mechanical trace represents the response associated with the propagated impulse induced by the current pulse. The current intensities used were 2 (left) and $4 \mathrm{~mA}$ (right). $21^{\circ} \mathrm{C}$.

portion of the nerve surface in the middle pool (see the upper trace), there was a gradual increase in the distance between the light-reflecting target on the nerve and the tip of the Fotonic sensor. At current intensities roughly 5-10 times the threshold for excitation, the smooth movement of the target was interrupted by a mechanical response associated with the production of a propagated action potential (Note that swelling of the nerve decreases the distance between the target and the sensor). With pulses of strong currents directed outwards through the nerve surface, a sharp mechanical response representing the initiation of a nerve impulse was superposed on a small and smooth mechanical change. There was a small difference in latency between the sharp mechanical response generated by an inward current and that of the response produced by an outward current. This difference is attributable to the involvement of a finite conduction time in excitation with an inward current (Note that, when the applied current is inwardly directed through the nerve surface in the middle pool, the nerve impulse is generated in the portion of the nerve in the lateral pools where the membrane current is outwardly directed). The results obtained with a Gulton bender were essentially the same as those described above.

Using the same experimental setup, the behavior of the nerve treated with tetrodotoxin (TTX) was examined. When TTX was added to the external medium at a concentration of $20-40 \mathrm{nM}$, these was a gradual reduction in amplitude of the 


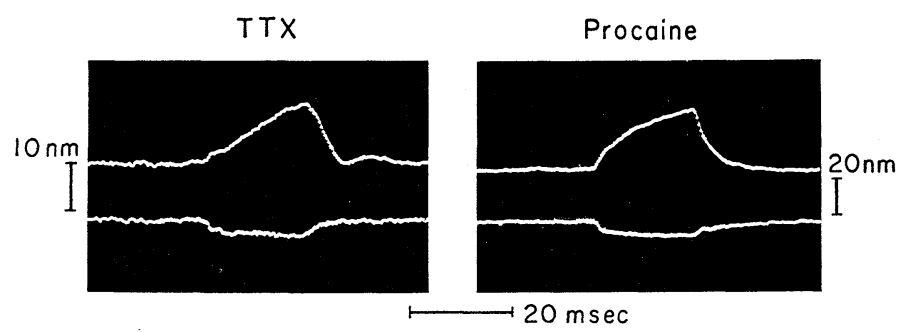

Fig. 4. Movements of the nerve surface produced by long pulses of electric currents applied to crab nerves treated with $40 \mathrm{~nm}$ tetrodotoxin (TTX) or with $4 \mathrm{~mm}$ procaine. The experimental setup illustrated in Fig. 3, top, was used. The current intensity used was $4 \mathrm{~mA} .22^{\circ} \mathrm{C}$.

sharp mechanical response. The record presented in Fig. 4, left, was obtained from a bundle of nerve fibers treated uniformly with $40 \mathrm{~nm}$ TTX. It is seen that TTX completely suppressed the sharp mechanical responses, which are accompanied by action potentials. The slow changes, on the contrary, were only slightly reduced by the powerful nerve poison.

The effects of local anesthetics were examined by the same procedure. The effect of $4 \mathrm{~mm}$ procaine was very similar to that of TTX (see Fig. 4, right).

\section{DISCUSSION}

The main objective of the experiments described under RESULTS was to clarify general physiological properties of the mechanical responses of the crustacean nerve. A small target placed on the surface of the nerve was shown to move transiently following electric stimulation. Usually the amplitude of the movement was $10-20 \mathrm{~nm}$ and was accompanied by a rise in pressure of $5 \mathrm{mg} / \mathrm{cm}^{2}$. The mechanical response was shown to travel along the nerve at a rate similar to the velocity of a propagated action potential. When nerve fibers were confined in a small slot, there was a rise in the pressure exerted by the nerve on the wall of the slot. Consequently, these mechanical responses of the nerve were interpreted as representing swelling of nerve fibers associated with generation of an action potential.

At the site of application of a long pulse of electric current, the mechanical response associated with initiation of a propagated nerve impulse was shown to be preceded by a small, continuous mechanical change. The mechanical responses associated with a propagated nerve impulse were suppressed by TTX, as well as by procaine. The continuous components of the mechanical change generated by electric currents were only slightly affected by anesthetics. Since substantially the same results were obtained by using a Gulton bender instead of a Fotonic sensor, the possibility was precluded that the observed results were produced by a direct effect of the applied current on the optical target. 
The ultimate goal of the present studies is to elucidate the macromolecular basis of the processes underlying the production of mechanical responses. It is well known that swelling of gels in inanimate colloidal materials is produced by invasion of water. Quite recently, we have obtained suggestive evidence that there is transport of water from outside when a mechanical response is produced in a crab nerve. We saw that the sum of the volume of the surrounding seawater and the nerve remains roughly unaltered when the nerve fibers were excited. Consequently, we are confronted with the following physiocochemical problem: how does a change in the membrane potential induce transport of water from the surrounding medium to the cortical layer of the protoplasm?

For studying this problem, the use of squid giant axons is undoubtedly more beneficial, because the membrane potential can be controlled more readily in giant axons than in crab nerve fibers. The results we have obtained so far indicate that the mechanical properties of the squid axon membrane are, in many respects, very similar to those of the crab nerve. The results of our studies on squid axons will be presented elsewhere.

\section{REFERENCES}

Bethe, A. (1920) Nervenpolarisationsbilder und Erregungstheorie. Pflügers Arch. Ges. Physiol., 183: 289-302.

Bryant, S. H. and Tobias, J. M. (1955) Optical and mechanical concomitants of activity in carcinus nerve. J. Cell. Comp. Physiol., 46: 71-95.

CoHEN, L. G. (1973) Changes in neuron structure during action potential propagation and synaptic transmission. Physiol. Rev., 53: 373-418.

FranK, G. M. (1958) Physical, chemical and structural processes during initiation and propagation of impulse through the nerve fiber. Izv. Akad. Nauk SSSR (Biol.), 23: 26-38.

Hill, B. C., Schubert, E. D., Nokes, M. A., and Michaelson, R. P. (1977) Laser interferometer measurement of changes in crayfish axon diameter concurrent with action potential. Science, 196: 426-428.

HILl, D. K. (1950) The volume change resulting from stimulation of a giant nerve fibre. $J$. Physiol. (Lond.), 111: 304-327.

IWASA, K. and TASAKI, I. (1980) Mechanical changes in squid giant axons associated with production of action potentials. Biochem. Biophys. Res. Commun., 95: 1328-1331.

Kayushin, L. P. and Lyudoovskaya, R. F. (1955) Elastic and electrical phenomena in the propagation of excitation. Dokl. Akad. Nauk SSSR, 102: 727-728.

Kornakova, E. V., Frank, G. M., and Steinhaus, L. N. (1947) On the structural processes in the nerve. Fiziol. Zh. SSSR, 33: 483-494.

Lettvin, J. Y., Sten-Knudsen, O., and Pitts, W. H. (1962) Mechanical responses in nerve. Quart. Prog. Rep. Mass. Inst. Technol., No. 64, 291-292.

SANDLIN, R., LERMAN, L., BARRY, W., and TASAKI, I. (1968) Application of laster interferometry to physiological studies of excitable tissues. Nature, 217: 575-576.

TASAKI, I. and IWASA, K. (1980) Shortening of nerve fibers associated with propagated nerve impulse. Biochem. Biophys. Res. Commun., 94: 716-720.

TASAKI, I. and SISCO, K. (1975) Electrophysiological and optical methods for studying the excitability of the nerve membrane. In: Methods in Membrane Biology, Vol. 5, ed. by Korn, E. D. Plenum Publ. Corp., New York, Chap. 4.

Vol. 30, No. 6, 1980 